\title{
Uncinate Preserving Procedures: A Leading Step toward Conservation in Functional Endoscopic Sinus Surgery
}

\author{
${ }^{1}$ Ashok K Gupta, ${ }^{2}$ Sourabha K Patro
}

\begin{abstract}
Uncinate process is thought to be having role in controlling airflow dynamics at the osteo meatal complex. Excision of uncinate process during traditional functional endoscopic sinus surgery (FESS) affects these airflow patterns increasing the flow in the sinuses in inspiration and decreasing it during expiration. Conventional way of performing a FESS always includes uncinectomy. However, preservation of uncinate process during surgery leads to the protection of the opened sinuses and avoid the attack of direct airflow to the sinus along with maintaining the normal nasal drainage from the sinus cavities. There is no clear evidence or consensus in the issue and regarding the feasibility of successful access in the ethmoids and sphenoids and beyond by preserving this immensely important land mark.

In this study, we have tried to see that whether it is feasible to conserve uncinate as a future eland mark and preserve more normal nasal physiology compared with the resection of uncinate process.
\end{abstract}

Keywords: FESS, Landmark, Outcome, Preservation, Uncinate process.

How to cite this article: Gupta AK, Patro SK. Uncinate Preserving Procedures: A Leading Step toward Conservation in Functional Endoscopic Sinus Surgery. Clin Rhinol An Int J 2015;8(1):12-14.

\section{Source of support: Nil}

Conflict of interest: None

\section{INTRODUCTION}

Uncinate process is a thin sickle shaped process in the middle meatus between inferior and middle turbinates. This has been considered as a part of the osteomeatal complex and thought to be having role in controlling local airflow dynamics. It is thought to maintain and restrict the maxillary and anterior ethmoidal airflow to

\footnotetext{
${ }^{1}$ Professor, ${ }^{2}$ Senior Resident

1,2Department of Otolaryngology and Head and Neck Surgery Postgraduate Institute of Medical Education and Research Chandigarh, India

Corresponding Author: Ashok K Gupta, Professor Department of Otolaryngology and Head and Neck Surgery Postgraduate Institute of Medical Education and Research Chandigarh, India, Phone: 09914209763, e-mail: drashokpgi@ hotmail.com
}

the desired levels in its normal physiological role. ${ }^{1}$ Excision of uncinate process during traditional functional endoscopic sinus surgery (FESS) affects these airflow patterns increasing the flow in the sinuses in inspiration and decreasing it during expiration with overall increase in the air flow velocities in the common meatus and the inferior meatus. ${ }^{2,3}$ This decrease in the sinus ventilation associated increased flow rates in the area of uncinate process leads to more gas exchange in the maxillary sinus compared to nasal cavities with preserved uncinate processes. And this increased gas exchange and decreased ventilation leads to accumulation of more amounts of non-oxygen gasses in the sinuses.

Conventional way of performing a FESS always includes uncinectomy. However, preservation of uncinate process during surgery leads to the protection of the opened sinuses and avoid the attack of direct airflow to the sinus along with maintaining the normal nasal drainage from the sinus cavities. ${ }^{4}$ The distance between uncinate process and the orbital wall is highly varable. Uncinate process helps as a major land mark in planning and performing endoscopic sinus surgery and while performing uncinectomy there is always an inherent risk of causing inadvertent injury to the orbit. ${ }^{5}$

When the surgical process does not involve any work near or in the maxillary sinus, we need to question ourselves that are we justified in doing an uncinectomy. There is no clear evidence or consensus in the issue and regarding the feasibility of successful access in the ethmoids and sphenoids and beyond by preserving this immensely important land mark.

Hence, we tried to address this issue in our study to see whether it is feasible to conserve uncinate as a future eland mark and preserve more normal nasal physiology compared with the resection of uncinate process. A leading step towards convervative approaches in FESS.

\section{MATERIALS AND METHODS}

All cases of sinunasal, orbital and optic nerve pathology, where the disease or the area of surgical interest was restricted to posterior ethmoids, sphenoid or beyond with grossly normal anterior ethmoidal gallery with no disease in the maxillary sinus, who presented or referred 
to the department of otolaryngology and head and neck surgery of our tertiary care institute between July 2012 and December 2013 were included in the study. All cases underwent detailed clinical examination and other head and neck examination as per routine institute protocol. These patients underwent contrast enhanced CT scans along with routine investigations for general anesthesia. Patients planned for optic nerve and orbital procedures were taken for MRI of orbit with $1 \mathrm{~mm}$ optic nerve cuts, visual evoked potentials, fundoscopy and field charting in cooperative patients. In all post-trauma cases neurosurgery clearance was taken before proceeding for surgery to rule out other intracranial pathologies which may have of higher significance or may adversely affect the result of surgery. All these patients were taken for surgery under hypotensive general anesthesia.

\section{RESULTS}

A total of 33 procedures were done by uncinate preserving technique during this period. These included nine optic nerve decompressions performed in cases of post-traumatic optic neuropathies, four optic nerve fenestration procedures performed in cases benign intracranial hypertension, six endoscopic medial orbital decompressions done in dysthyroid orbitopathy, seven cases of endoscopic repair of CSF rhinorrhea, five biopsy procedures performed in suspected cases of invasive aspergillosis and one procedure each perfomed in cases of meningioma of sphenoid sinus and fungal ball of sphenoid sinus.

Uncinectomy was avoided in all these procedures and so also a middle meatal antrostomy and an ethmoidectomy was done by initiating with the step of uncapping of bulla then followed by post-ethmoidectomy and sphenoidotomy and further steps as required as per the procedure performed. All these patients are under our continuous follow-up after these procedures.

All these procedures were performed by a single surgeon, the lead author. There was no difficulty in accessing ethmoids and further even without uncinectomy. All these cases antrostoma could not be used as a landmark, however, all other landmarks were being present and there was no hindrance in the prodcedure.

Preservation of uncinate led to preservation of osteomeatal complex. Each patient was analyzed in the postoperative period to see for the changes in the nasal cavity in nasal endoscopy and changes in the osteomeatal complex was observed. Any discharge or pus present in the middle meatus or coming from the hiatus semilunaris was checked to see for the status of the maxillary sinus and osteomeatal complex. All these cases, it could be observed that there was no discharge or crust in the osteomeatal complex area. The sinuses were healthy and all patients showed a Kupferberg's nasal endoscopy grade ' 0 '.

In the follow-up of 6 months from the date of surgery, inadequate drainage of the sinuses as noted by presence of pool of secretions, could not be seen in any case where uncinate was preserved. Condition of the mucosa was normal without any edema or polypoidal change. In all these cases uncinate process maintained its normal anatomical shape in follow-up.

Comparison of the sinunasal quality of life between cases with intact uncinate and cases with uncinectomy did not reveal any significant difference $(p>0.05)$. These control cases included retrospective record review of equal number of randomly selected similar cases performed by the senior surgeon during the same time period, where uncinectomy was done.

\section{DISCUSSION}

Conservative approaches in endoscopic sinus surgery are always been sought for. This is to ensure a good functional outcome. The persistence of allergic nasal discharge and post-nasal discharge after FESS is thought to be due to persistence of exposure of the ethmoid and maxillary air cells to allergens, which occurs in the absence of uncinate process. ${ }^{6}$ It has also been noted that the airflow pattern changes in after uncinectomy ${ }^{2}$ and there occurs increased antigen exposure of the sinus mucosa. $^{7}$

Our aim of performing this study was to look for the feasibility of this approach and to look for the postoperative cavity conditions in order to take a step further in the process of conservation in endoscopic sinus surgery (Figs 1A and B).

Comparison of the sinunasal quality of life between cases with intact uncinate was similar to those with uncinectomy. Uncinate process when preserved protects the maxillary and ethmoid sinuses from continuous exposure to allergens and repeated insult to the sinus mucosa. Literature review shows evidences of maxillary sinus hypoplasia to be associated with the absence of uncinate process. Bolger et $\mathrm{al}^{8}$ described this association of maxillary sinus hypoplasia with hypoplasia of uncinate process in congenital cases and similar association was also shown to be associated in acquired cases by Kosko et al. ${ }^{9}$ This association in acquired cases is thought to be due to increased exposure of the sinus mucosa to allergens. ${ }^{7}$ 

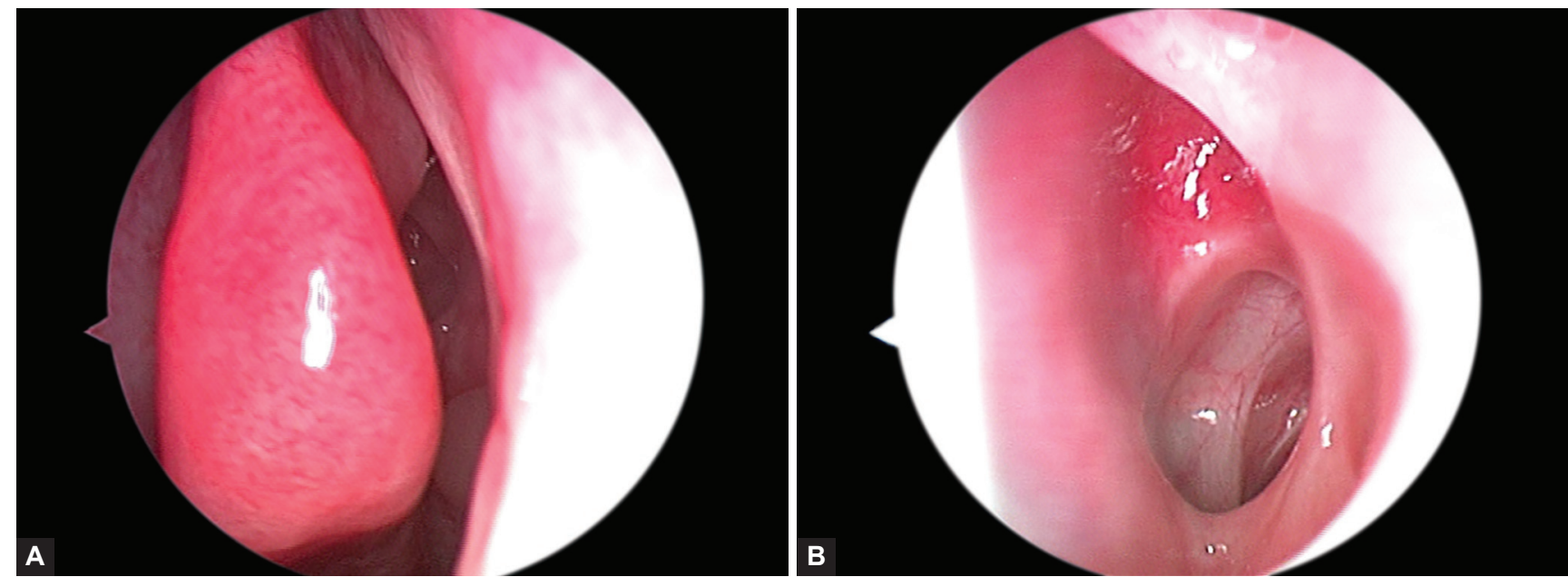

Figs 1A and B: Postoperative status of the nasal cavity in the follow-up after an uncinate preserving procedure. (sphenoethmoidectomy done with intact uncinate process)

With the resection of uncinate process, maxillary sinus, frontal recess and part of bulla and anterior ethmoids remains exposed to the turbulent inspiratory airflow and increased load of allergens as compared to the normal ventilation of these sinuses during the expiratory phase as seen in the presence of uncinate process. Hence we postulate that the presence of normal healthy sinus mucosa and maintenance of physiological drainage and protection of repeated insult to the sinus mucosa by the inspiratory turbulent currents and allergens could have been prevented with preservation of the uncinate process.

\section{CONCLUSION}

This proposed surgical technique of uncinate preservation is still experimental and is under evaluation. We could observe normal sinus mucosal condition in the nasal endoscopy in the follow-up. The sinunasal quality of life was also similar to the cases with uncinate resection. Hence we propose that even though it didn't show any statistically significant advantage over the cases with uncinectomy, the advantage of preservation does include protection of sinus mucosa to repeated insult and maintenance near normal anatomy and physiology. Hence, we advocate this technique as a proposed way to preserve near normal physiology in terms of mucosal drainage of the operated nasal cavities necessitating and inviting further research. This could be a better technique in cases of pathologies not involving the maxillary sinus, intact uncinate process and where the area of surgical interest is posterior ethmoids or beyond.

\section{REFERENCES}

1. Jiang GL, Xu G. Change of airflow patterns inside the maxillary sinus influenced by uncinate process. Zhonghua er bi yan hou tou jing wai ke za zhi $=$ Chinese journal of otorhinolaryngology head and neck surgery 2009;44(6):460-463.

2. Xiong GX, Li JF, Zhan JM, Jiang GL, Rong LW, Xu G. Influence of uncinate process on aerodynamic characteristics of nasal cavity and maxillary sinus. Zhonghua er bi yan hou tou jing wai ke za zhi $=$ Chinese journal of otorhinolaryngology head and neck surgery 2009;44(1):10-15.

3. Xiong GX, Zhan JM, Zuo KJ, Rong LW, Li JF, Xu G. Use of computational fluid dynamics to study the influence of the uncinate process on nasal airflow. J Laryngol Otology 2011; 125(1):30-37.

4. Xu G. Preservation or reconstruction of uncinate process in endoscopic sinus surgery. Zhonghua er bi yan hou tou jing wai ke za zhi $=$ Chinese journal of otorhinolaryngology head and neck surgery 2007;42(1):3-6.

5. Hopkins C, Slack R, Lund V, Brown P, Copley L, Browne J. Long-term outcomes from the English national comparative audit of surgery for nasal polyposis and chronic rhinosinusitis. The Laryngoscope 2009;119(12):2459-2465.

6. Kennedy DW, Zinreich SJ, Rosenbaum AE, Johns ME. Functional endoscopic sinus surgery: theory and diagnostic evaluation. Arch Otolaryngol 1985;111(9):576-582.

7. Nayak DR, Balakrishnan R, Murty KD. Functional anatomy of the uncinate process and its role in endoscopic sinus surgery. Indian journal of otolaryngology and head and neck surgery: official publication of the Association of Otolaryngologists of India 2001;53(1):27-31.

8. Bolger WE, Woodruff WW Jr, Morehead J, Parsons DS. Maxillary sinus hypoplasia: classification and description of associated uncinate process hypoplasia. Otolaryngologyhead and neck surgery: Official Journal of American Academy of Otolaryngology—Head and Neck Surgery 1990; 103(5 pt 1):759-765.

9. Kosko JR, Hall BE, Tunkel DE. Acquired maxillary sinus hypoplasia: a consequence of endoscopic sinus surgery? Laryngoscope 1996;106(10):1210-1213. 